\title{
Association between the extent of white matter damage and early cognitive impairment following acute ischemic stroke
}

\author{
JIAN LI $^{1}$, YONG ZHAO ${ }^{1}$ and JINYING MAO ${ }^{2}$ \\ ${ }^{1}$ Department of Emergency, Neurology Division, and ${ }^{2}$ The Second Department of Cardiology, \\ Weifang People's Hospital, Weifang, Shangdong 261041, P.R. China
}

Received May 30, 2016; Accepted December 30, 2016

DOI: $10.3892 /$ etm.2017.4035

\begin{abstract}
White matter (WM) injury following acute ischemic stroke (AIS) is associated with cognitive decline. Establishing relationships between the specific cognitive tests used to assess post-AIS cognition and various clinical indices of WM injury severity and distribution may aid in prognosis and early treatment decisions. We enrolled 62 patients with AIS to Weifang People's Hospital between September 2014 and August 2015. WM lesion severity and distribution were examined by computed tomography (CT) and magnetic resonance imaging (MRI). The Blennow scale was used for scoring the distribution and degree of WM lesions (WMLs) on CT images, the Fazekas scale for scoring periventricular and deep WMLs on MRI, and the Cholinergic Pathways Hyperintensities Scale (CHIPS) for scoring MRI manifestation of cholinergic fiber damage. The 8-domain Montreal Cognitive Assessment (MoCA) was used to evaluate cognitive function. Mean \pm standard deviation scores on the Blennow scale was $1.6 \pm 0.5$; Fazekas scale, $3.4 \pm 0.8$; and CHIPS, $65.7 \pm 12.5$. The proportion of patients with a MoCA score $<26$ (indicating cognitive dysfunction) was significantly higher in subgroups with Blennow scale score $>2$, Fazekas scale score $>4$, and CHIPS score $>51$ (all $\mathrm{P}<0.001$ ). The MoCA score was negatively correlated with Blennow scale score $(\mathrm{r}=-0.326$, $\mathrm{P}=0.002)$, Fazekas scale score ( $\mathrm{r}=-0.404, \mathrm{P}=0.031)$, and CHIPS score $(\mathrm{r}=-0.234, \mathrm{P}=0.042)$. Thus, the degree and distribution of whole-brain, deep, and cholinergic WMLs were associated with cognitive impairment. The Blennow scale, Fazekas scale, and CHIPS all provide good predictive efficacy of post-AIS cognitive dysfunction.
\end{abstract}

Correspondence to: Ms. Jinying Mao, The Second Department of Cardiology, Weifang People's Hospital, 151 Guangwen Street, Weifang, Shangdong 261041, P.R. China

E-mail:mao_jinying9@163.com

Key words: acute ischemic stroke, white matter lesions, cognitive impairment, Blennow scale, Fazekas scale, cholinergic pathway scale, Montreal Cognitive Assessment

\section{Introduction}

Acute ischemic stroke (AIS) usually causes focal ischemia/necrosis and more widespread apoptosis, resulting in high incidences of permanent disability and mortality (1). White matter (WM) lesions (WMLs) caused by axon loss and demyelination due to chronic ischemia or hypoperfusion of the brain often result in long-term cognitive impairment (2). While the pathogenic mechanisms for WMLs may be similar across WM pathways, the clinical manifestations differ markedly (3). Thus, the measured severity of cognitive decline is highly dependent on the breadth of the cognitive battery employed. The Montreal Cognitive Assessment (MoCA) is a multi-domain scale for rapidly identifying cognitive impairment. There are numerous rating scales to evaluate the severity and distribution of WMLs from neuroimaging. The Blennow scale is used for analyzing and assessing the degree and distribution of WMLs on computed tomography (CT) (4), and the Fazekas scale is used for analyzing and assessing periventricular and deep WMLs on magnetic resonance (MR) imaging (MRI) (5). The Cholinergic Pathways Hyperintensities Scale (CHIPS) was developed for semi-quantitative evaluation of cholinergic pathway loss on MRI (6). It is critical to identify the severity and extent of cognitive impairment for timely and effectively intervention. This study examined the relationship between post-AIS cognitive dysfunction, as evaluated by the MoCA, and the severity and distribution of WMLs, evaluated using the Blennow scale, Fazekas scale, and CHIPS.

\section{Materials and methods}

Patients. Sixty-two AIS patients admitted to Weifang People's Hospital (Shangdong, China) between September 2014 and August 2015 were selected using computer-generated random number matching for this study. The final cohort consisted of 34 males and 28 females between 45 and 80 years (mean, $58.7 \pm 8.8$ years). Inclusion criteria were as follows: i) Meeting the Chinese and WHO AIS diagnosis and treatment criteria, with MRI and CT confirmation; ii) onset $\leq 7$ days before examination; and iii) able to complete research project and the MoCA. Exclusion criteria were as follows: i) Brain trauma, brain tumor, cerebral aneurysm, cerebrovascular developmental malformation, cerebral hemorrhage, and other craniocerebral 
diseases; ii) underlying diseases, such as heart, lung, kidney, or liver dysfunction; iii) severe condition predictive of $<12$-month survival; and iv) pre-existing cognitive impairment due to Alzheimer's disease or other neuropsychiatric diseases. This study was approved by the Weifang People's Hospital Ethics Committee, and informed consent was obtained from all the patients or their relatives.

$C T$. Patients underwent brain CT scan with a scanning seam thickness of $5 \mathrm{~mm}$ and layer spacing of $5 \mathrm{~mm}$. After injection of the contrast medium, CT scanning of the medial temporal lobe, bilateral frontopolar lobes, parietal lobe, and basal ganglia was performed.

MRI. Patients underwent 3.0-T MRI in the supine position with eyes closed. Patients wore earplugs to reduce scanning noise, and head motion was restricted using foam pads. Patients were requested to keep quiet during scans, including T1-weighted, T2-weighted, and FLAIR sequences, to assess WML severity/location and to exclude other encephalopathies. Resting-state functional MRI was conducted using the echo-planar imaging (EPI) sequence (seam thickness, $3 \mathrm{~mm}$; axial view, 36 layers; voxel size, $3.5 \times 3.5 \times 3.0 \mathrm{~mm}^{3}$; matrix, $64 \times 64$; repetition time (TR), 2,000 $\mathrm{msec}$; echo time (TE), $30 \mathrm{msec}$; flip angle (FA), 90'; field of view (FOV), 222×222 mm). High-resolution T1-weighted structural images were collected using a 3D MP-RAGE sequence (seam thickness, $1 \mathrm{~mm}$; sagittal view, 176 layers; voxel size, $1.0 \times 1.0 \times 1.0 \mathrm{~mm}^{3}$; matrix, 256x256; TR, 1,900 msec; TE $2.52 \mathrm{msec}$; inversion time (TI), $900 \mathrm{msec}$; FA, $9^{\circ}$; FOV, 256x256 mm).

Image processing. CT images were imported into a processing station. MRI data were processed by SPM8 running on the MATLAB R2012a platform. Post-processing of MR images included time correction, dynamic correction, high-resolution structural image segmentation, regression removal of whole-brain signals, space standardization, spatial smoothing, de-systematic linear drift correction, band-pass filtering, and standardized calculation of amplitude of low-frequency fluctuation (ALFF) value.

MoCA. The MoCA evaluates 8 cognitive domains: Visuospatial/executive function, memory, naming, language, attention, delayed recall, abstract thinking, and orientation. The total score is 30 points and a score of 26 or lower is indicative of cognitive impairment.

Blennow scale. Lesions were scored from 0 to 3 according to the following patterns: i) No reduced WM signals (0 points); ii) reduced WM signals in occipital angle and the edge of frontal angle of lateral ventricles (1 point); iii) reduced WM signals in occipital angle surroundings and the frontal angle of lateral ventricles with extension to the semioval center ( 2 points); and iv) reduced WM signals around the whole lateral ventricles and extending to the semioval center with blending (3 points). Lesion severity was scored according to the following changes in signal strength: i) No reduction in signal ( 0 point); ii) mildly reduced signals (1 point); iii) moderately reduced signals ( 2 points); and iv) substantially reduced signals (3 points).
Fazekas scale. Periventricular lesions were scored according to the following patterns: i) No lesions (0 points); ii) pencil-like or cap-like thin lesions (1 point); iii) smooth haloes at lesion site ( 2 points); and iv) irregular periventricular high signals extending to deep WM (3 points). Deep WMLs were scored according to the following patterns: i) No lesions (0 points); ii) punctate separate lesions (1 point); iii) fused lesions (2 points); and iv) large fused lesions (3 points). The total score was obtained by adding the individual periventricular and deep WML scales together.

CHIPS. Measurement zones within four WM regions were demarcated on axial T2-weighted images of the lateral ventricles and the third ventricle: i) The semioval center (4 separate zones: Bilateral anterior and posterior zones); ii) corona radiata (6 separate zones: Bilateral anterior, posterior, and cingulate gyrus zones); iii) high external capsule layer (6 separate zones: Bilateral anterior, posterior, and cingulate gyrus zones); and iv) low external capsule layer (4 separate zones: Bilateral anterior and posterior zones). The WML severity in each zone was scored as: i) Normal (0 points); ii) $<50 \%$ of region involved (1 point); and iii) $>50 \%$ of region involved (2 points). Severity scores were weighted based on cholinergic fiber distribution density. The weighting coefficients were 1 for the semioval center, 2 for the corona radiata, 3 for the high external capsule layer, and 4 for the low external capsule layer. The score for each region was obtained by adding all points for the measurement of small zones and multiplying by the weighting coefficient. The total score was calculated as the sum of scores for all four regions (maximum final score of 100 , with 50 points/hemisphere).

Statistical analysis. SPSS 19.0 software was used for data processing. Measurement data with normal distributions are expressed as mean \pm standard deviation (SD). Group means were compared by t-test. Enumeration data are expressed by case number $(\%)$. Proportions were compared by $\chi^{2}$ test. The strengths of correlations were assessed by Spearman's correlation analysis. A $\mathrm{P}<0.05$ was considered to indicate a statistically significant difference.

\section{Results}

Correlation of MoCA scores with Blennow scale scores. The mean Blennow scale score was $1.6 \pm 0.5$ (maximum 3 ) for all 62 subjects, of which 33 were scored as 0 or 1 and 29 as 2 or 3 (indicative of more severe and extensive WMLs). MoCA score was significantly higher in the less severe $0-1$ point Blennow scale group compared to the 2-3 points group, and the proportion of subjects scoring $<26$ points on the MoCA (defined as cognitive dysfunction) was significantly higher in the 2-3 points group $(\mathrm{P}=0.034)$ (Table I). The MoCA score was negatively correlated with Blennow scale score $(r=-0.326$, $\mathrm{P}=0.002)$.

Correlation of MoCA scores with Fazekas scale scores. The mean Fazekas scale score for periventricular plus deep WMLs was 3.4 \pm 0.8 (maximum 6) for the entire 62-patient cohort, of which 30 patients scored 0-3 and 32 scored $4-6$ points. Again, MoCA score was significantly higher in the 0-3 points Fazekas 
Table I. Association of MoCA score with Blennow scale score.

\begin{tabular}{lccc}
\hline Groups & Case no. & MoCA score & $<26$ points, $\mathrm{n}(\%)$ \\
\hline 0-1 point & 33 & $29.3 \pm 4.5$ & $6(18.2)$ \\
2-3 points & 29 & $24.5 \pm 5.3$ & $23(79.3)$ \\
t-test & & 5.320 & \\
$\chi^{2}$ & & & 23.168 \\
P-value & & 0.034 & $<0.001$ \\
\hline
\end{tabular}

MoCA, Montreal Cognitive Assessment.

Table II. Association of MoCA score with Fazekas scale score.

\begin{tabular}{lccc}
\hline Groups & Case no. & MoCA score & $<26$ points, $\mathrm{n}(\%)$ \\
\hline 0-3 points & 30 & $28.6 \pm 6.3$ & $7(23.3)$ \\
4-6 points & 32 & $25.6 \pm 6.2$ & $24(75.0)$ \\
t-test & & 5.237 & 16.533 \\
$\chi^{2}$ & & & $<0.001$ \\
P-value & & 0.036 & \\
\hline
\end{tabular}

MoCA, Montreal Cognitive Assessment.

scale group (with less severe and extensive WMLs), and the proportion of patients with cognitive dysfunction according to MoCA score $<26$ points was significantly higher in the 4-6 points Fazekas scale group with more severe periventricular/deep WMLs $(\mathrm{P}<0.01)$ (Table II). In addition, MoCA score was also negatively correlated with the Fazekas scale score $(\mathrm{r}=-0.404, \mathrm{P}=0.031)$.

Correlation of MoCA and CHIPS scores. The mean CHIPS score (maximum 100) was $65.7 \pm 12.5$ for all 62 subjects, of which 25 scored 0-50 points and 37 scored 51-100 points. MoCA score was significantly higher in the $0-50$ points group than in the 51-100 points group, and the proportion with cognitive impairment (MoCA score $<26$ points) was significantly higher in the 51-100 points CHIPS group $(\mathrm{P}<0.05)$ (Table III). MoCA score was negatively correlated with CHIPS score $(\mathrm{r}=-0.234, \mathrm{P}=0.042)$.

\section{Discussion}

WMLs following AIS appear as hyperintense regions on FLAIR and T2-weighted MR images, which has been demonstrated to be an independent risk factor of dementia. The mechanisms and etiology of WML are not completely clear but appear to differ from those involved in ischemic gray matter damage (7). Moreover, differences in diagnostic criteria, time after onset, cognitive tests used, analysis methods, and patient heterogeneity have obscured the relationships between specific cognitive deficits and WML pattern (8). We show that the severity of cognitive dysfunction is associated with the general severity and extent of WMLs. Cognitive dysfunction may arise from disruption of prefrontal-subcortical pathways, various
Table III. Association of MoCA score with CHIPS score.

\begin{tabular}{lccc}
\hline Groups & Case no. & MoCA score & $<26$ points, $\mathrm{n}(\%)$ \\
\hline $0-50$ points & 25 & $30.2 \pm 7.5$ & $5(20.0)$ \\
$51-100$ points & 37 & $23.9 \pm 8.0$ & $25(67.6)$ \\
$\mathrm{t}$ & & 6.231 & \\
$\chi^{2}$ & & & 13.517 \\
P-value & & 0.025 & $<0.001$ \\
\hline
\end{tabular}

MoCA, Montreal Cognitive Assessment; CHIPS, Cholinergic Pathways Hyperintensities Scale.

corticocortical pathways, and/or global regulatory systems such as the cholinergic system $(9,10)$. Cholinergic pathway was distributed from the basal ganglia to most regions of the cortex. Given that most of its fibers were unmyelinated, it was sensitive to vascular injury. Electrophysiological research and animal studies have demonstrated that cholinergic pathway mainly regulates cognitive processing, learning and memory secondly.

Slowing the progression of WMLs following AIS may therefore mitigate the severity of cognitive impairment. However, this requires not only early detection of WMLs but also greater knowledge of the precise relationships between WML patterns and specific cognitive impairments.

The distribution and degree of WMLs are assessed on $\mathrm{CT}$, and scored using the Blennow scale, where the final score is a mean value $(11,12)$. Periventricular and deep WMLs are seen as hyperintensities on T2-weighted MR images and are scored separately using the Fazekas scale, where the total score $(13,14)$ reflects the sum of both types of WM injury. Cholinergic nerve fiber distribution in WM was not determined until the 1990s on MRI. Since that time, CHIPS has proven highly effective for predicting cognitive impairments caused by cholinergic WMLs. Both Fazekas and CHIPS are based on MRI, however, with different emphasis in evaluation of WMLs. CHIPS was more specific in evaluation of the damage of cholinergic pathway, thus reflects the extent of cognitive disorders. However, given that CHIPS only focuses on cholinergic fibers emitted from nucleus basalis of Meynert, not all cholinergic pathway of cognitive process, it has some limitations in evaluation of cognitive disorders.

In our study, all WML assessments were significantly correlated with cognitive dysfunction, as assessed by the MoCA. Thus, combined evaluation of damage to the cholinergic pathway, periventricular and deep WM, and semioval center may reveal the bases for specific cognitive domain impairments $(15,16)$.

The MoCA is a simple screening tool that assesses dysfunction in multiple cognitive domains (17). Various studies have showed that MoCA was more effective in evaluating cognitive functions, such as alternate connection, attention, language fluency, abstract cognitive domain. In this study, patients scoring below 26 were much more likely to exhibit severe and/or extensive WMLs as assessed by the Blennow scale, the Fazekas scale, and CHIPS, underscoring the importance of WMLs in post-AIS cognitive dysfunction. Further, all scale 
scores had significant negative correlation with MoCA scores, indicating reciprocal predictive efficacy that may illuminate the cognitive functions of specific WM tracts.

In conclusion, the severity and extent of WMLs in AIS is strongly associated with the severity of cognitive impairment. Moreover, Blennow scale, Fazekas scale, and CHIPS scores are predictive of cognitive impairment, as assessed by the MoCA. Future studies are warranted to assess correlations of these WML metrics with deficits in specific cognitive domains.

\section{References}

1. Huang Q, Song HQ, Ji XM, Cheng WY, Feng J, Wu J and Ma QF: Generalization of the right acute stroke prevention strategies in reducing in-hospital delays. PLoS One 11: e0154972, 2016.

2. Leonards CO, Ipsen N, Malzahn U, Fiebach JB, Endres M and Ebinger M: White matter lesion severity in mild acute ischemic stroke patients and functional outcome after 1 year. Stroke 43 3046-3051, 2012.

3. Liao J, Zhu Y, Zhang M, Yuan H, Su MY, Yu X and Wang H: Microstructural white matter abnormalities independent of white matter lesion burden in amnestic mild cognitive impairment and early Alzheimer disease among Han Chinese elderly. Alzheimer Dis Assoc Disord 24: 317-324, 2010.

4. Curtze S, Melkas S, Sibolt G, Haapaniemi E, Mustanoja S, Putaala J, Sairanen T, Tiainen M, Tatlisumak T and Strbian D: Cerebral computed tomography-graded white matter lesions are associated with worse outcome after thrombolysis in patients with stroke. Stroke 46: 1554-1560, 2015.

5. Maier O, Wilms M, von der Gablentz J, Krämer UM, Münte TF and Handels H: Extra tree forests for sub-acute ischemic stroke lesion segmentation in MR sequences. J Neurosci Methods 240: 89-100, 2015.

6. Behl P, Bocti C, Swartz RH, Gao F, Sahlas DJ, Lanctot KL, Streiner DL and Black SE: Strategic subcortical hyperintensities in cholinergic pathways and executive function decline in treated Alzheimer patients. Arch Neurol 64: 266-272, 2007.

7. Fern RF, Matute C and Stys PK: White matter injury: ischemic and nonischemic. Glia 62: 1780-1789, 2014.
8. Yamano S, Horii M, Takami T, Sakuma M, Morimoto T, Okada S, Taoka T, Kichikawa K, Sasamura H, Itoh H, et al: Comparison between angiotensin-converting enzyme inhibitors and angiotensin receptor blockers on the risk of stroke recurrence and longitudinal progression of white matter lesions and silent brain infarcts on MRI (CEREBRAL study): rationale, design, and methodology. Int J Stroke 10: 452-456, 2015.

9. Bolandzadeh N, Davis JC, Tam R, Handy TC and Liu-Ambrose T: The association between cognitive function and white matter lesion location in older adults: a systematic review. BMC Neurol 12: 126, 2012.

10. Yan T, Yu JR, Zhang YP and Li T: Analysis on correlation of white matter lesion and lacunar infarction with vascular cognitive impairment. Int J Clin Exp Med 8: 14119-14122, 2015.

11. Tu Q, Ding B, Yang X, Bai S, Tu J, Liu X, Wang R, Tao J, Jin H, Wang Y, et al: The current situation on vascular cognitive impairment after ischemic stroke in Changsha. Arch Gerontol Geriatr 58: 236-247, 2014

12. Li L, Simoni M, Küker W, Schulz UG, Christie S, Wilcock GK and Rothwell PM: Population-based case-control study of white matter changes on brain imaging in transient ischemic attack and ischemic stroke. Stroke 44: 3063-3070, 2013.

13. Boutet C, Rouffiange-Leclair L, Schneider F, Camdessanché JP, Antoine JC and Barral FG: Visual assessment of age-related white matter hyperintensities using FLAIR images at 3 T: interand intra-rater agreement. Neurodegener Dis 16: 279-283, 2016.

14. Obara T, Hasegawa H, Wachi Y, Tanaka M, Sato M, Kobayashi Y, Koshiba H, Nagai K, Yamada Y, Matsui T, et al: The characteristics of a comprehensive geriatric assessment in patients with mild cognitive impairment with a cerebral white matter lesion. Nippon Ronen Igakkai Zasshi 52: 399-410, 2015 (In Japanese).

15. Helenius J, Goddeau RP Jr, Moonis M and Henninger N: Impact of leukoaraiosis burden on hemispheric lateralization of the National Institutes of Health Stroke Scale Deficit in acute ischemic stroke. Stroke 47: 24-30, 2016.

16. McNeely AA, Ramirez J, Nestor SM, Zhao J, Gao F, Kiss A, Stuss DT and Black SE: Cholinergic subcortical hyperintensities in Alzheimer's disease patients from the Sunnybrook Dementia Study: relationships with cognitive dysfunction and hippocampal atrophy. J Alzheimers Dis 43: 785-796, 2015.

17. Mi T, Qu CQ, Wang X, Sun QJ, Sun H and Du YF: Characteristics of cognitive impairment of different infarct locations among patients after acute ischemic stroke. Zhonghua Yi Xue Za Zhi 96: 1205-1207, 2016 (In Chinese). 